\title{
Spatial Scheduling in Multiuser Wireless Systems: From Power Allocation to Admission Control
}

\author{
Diego Bartolomé and Ana I. Pérez-Neira
}

\begin{abstract}
Given a zero forcing transmit beamforming, we focus on how the multi-antenna access point distributes the scarce resource (power) among the single-antenna terminals. Since there is a clear trade-off between the satisfaction of the individual needs and the global performance of the cell, several criteria are proposed, ranging from a classical physical layer point of view of capacity (rate) maximization to Bit Error Rate (BER)based cost functions, which are closer to the second layer of the protocol stack. Between two traditional techniques, namely the uniform power allocation and the equal BER and rate, a new one is proposed, which ultimately provides an intermediate performance. Then, we add BER (or Signal to Noise Ratio) constraints so that the admission control problem has to be solved. Among traditional options, we propose a new mechanism to balance the above-mentioned trade-off between the total performance and the particular user behavior. The results in terms of fairness are presented by a mean vs. variance plot and by the Gini plot.
\end{abstract}

Index Terms-Scheduling, smart antennas, fairness, power allocation.

\section{INTRODUCTION}

$\mathbf{T}$ HIS paper deals with the simultaneous downlink communication of a multi-antenna Base Station (BS) or Access Point (AP) with several single antenna terminals. In this case, the spatial domain is used to enhance the scheduling, which consists in the assignment of a certain rate to all the users, or in the denial of service if some minimum requirements cannot be fulfilled, i.e. the admission control. Basically, the scheduling consists of dividing the limited available resources among the active users. Instead of the link bandwidth, the scarce resource is the instantaneous output power, which is usually specified by regulatory authorities.

The solution of the scheduling at the physical layer (PHY) is divided into two steps, namely the transmit beamforming and the power allocation. As in [1], terminals are dumb, so that all the intelligence is located at the multi-antenna AP. The AP performs the transmit beamforming, so that the symbols the terminals receive are only corrupted by noise and not by the signals intended for the other users. Therefore, the terminals shall not perform any filtering, so that their computational load is reduced and their battery life time is increased. Besides,

Manuscript received October 6, 2003; revised July 12, 2005 and November 30, 2005; accepted November 30, 2005. The associate editor coordinating the review of this paper and approving it for publication was L.-C. Wang. Partially presented at ISSPA 2003 and at ICASSP 2004, this work has been supported by the CTTC, by the EU under IST-2001-38835, by the Spanish government under FIT-070000-2003-257, TIC2002-04594-C02-01, and by the Catalan grant 2003 FRI 00190.

D. Bartolomé is with the Technology Transfer Area at the Catalonian Telecommunications Technological Center (CTTC), Castelldefels, Spain (email: dbartolome@ieee.org).

A. I. Pérez-Neira is associate professor at the Technical University of Catalonia (UPC), Barcelona, Spain (email: anuska@gps.tsc.upc.es).

Digital Object Identifier 10.1109/TWC.2006.03520. they do not need to be aware of the channels from the other users. This seems a well-suited implementation for the Spatial Division Multiple Access (SDMA), since the resources granted for the users do not overlap [2]. The beamforming criterion that matches those requirements is Zero Forcing (ZF), which provides a low-complex closed-form solution to create parallel and orthogonal equivalent channels for the users that are being served, without own-cell interference [3]. Compared to optimum downlink beamforming [4], the main powerful characteristics are the low complexity and the closed-form solution. Moreover, it has been recently shown that if bit allocation algorithms are used, the performance of optimal downlink beamforming is equivalent to that of ZF [5].

Once the spatial architecture has been established, the AP has to distribute the available power among the users. Since there is a clear tradeoff between the satisfaction of the individual needs and the global performance of the cell [6], the fairness criterion determines the cost function of the problem. On the one hand, it is well-known that optimizing the global performance implies an asymmetric distribution of the resources, i.e. some are given more than others. On the other hand, max-min or min-max schemes [7] distribute the resources equally, i.e. the gains are cell-wide at the expense of loosing in global performance. In this paper, the authors concentrate on the instantaneous fairness, i.e. the implications of the resource allocation in the short-term in terms of mean performance and fairness. The authors propose a new technique, the Equal Proportional SNR (EPS) that provides an intermediate point between two traditional techniques, obtaining a good balance of the trade-off among the global performance and the individual needs.

Regarding fairness, two well-suited options to show it have been proposed in [2], namely, i) a mean vs. variance plot, and ii) the Gini plot as a measure of inequality. On the one hand, the former overcomes the relative nature of traditional indices, and plots the mean of the achieved performance metric ${ }^{1}$ vs. its variance among users. This shows the performance not only asymptotically but also for intermediate values, which are certainly the most reasonable. On the other hand, the Gini plot measures the degree of fairness of a resource allocation, see Fig. 1, which shows the percentage of the resource as a function of the percentage of the population. The perfect equality is the 45-degree line starting at the origin, i.e. for any percentage of the population (users) the resource is shared equally among all of them. The other curves correspond to different resource allocations reflecting that the income/resource share grows at much slower rate as the population share increases, thus there is a higher degree of resource concentration within the population [8]. Indeed, these two innovative plots are used

\footnotetext{
${ }^{1}$ The selected metrics in this paper are the rate and the BER.
} 


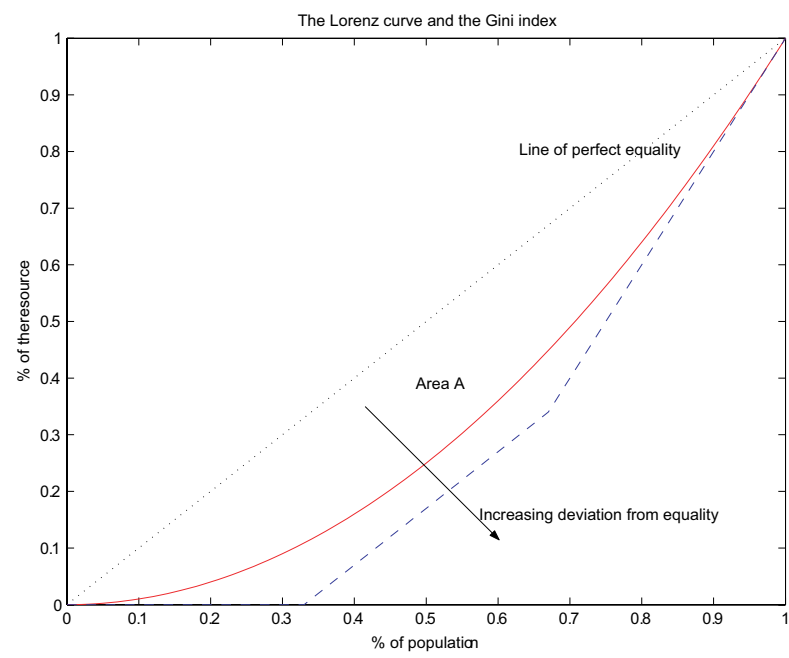

Fig. 1. The Gini plot: percentage of the resource as a function of the percentage of the population.

throughout the paper to show the performance of the proposed power allocation schemes in terms of fairness at the PHY.

Even with the optimal power allocation, it might happen that not all the active users can be scheduled. In this case, the multi-antenna BS has to decide which users are selected, that is the Spatial Admission Control (SAC) mechanism [9]. Additionally the SAC shall fulfill the QoS requirements of the users, e.g. in terms of BER or SNR. In the proposed SDMA system, the interactions among the users play a very important role and thus deeply impact on the selection of the users in the optimization of a certain criterion. Indeed, the performance varies significantly depending on the users that are being served, because more power is required if users with correlated channels are scheduled, see [2]. In this paper, we extend the power allocation criteria to deal with the admission control, analyze them in terms of diversity gain and multiplexing gain. Again, the EPS strategy is in between traditional options in the literature.

This paper is organized as follows after the problem statement that is presented next. In Section III, several power allocation techniques are proposed for this multi-antenna multi-user system, and the author evaluates the implications of the power allocation within the scheduling at both the PHY and the DLC, especially in terms of fairness. Then, section IV introduces a new power allocation strategy to better balance the trade-off between the global performance and the individual needs. Finally, in Section V, the admission control mechanisms are compared, just before the final conclusions. Simulations are given for each section.

\section{Problem Statement}

Boldface capital (lowercase) letters refer to matrices (vectors). The conjugate transpose of $\mathbf{a}$ is $\mathbf{a}^{H}$ and the element at row $i$ and column $j$ of $\mathbf{A}$ is denoted by $[\mathbf{A}]_{i, j}$. Unless indicated, the base-2 logarithm of $a$ is $\log (a)$, tr denotes the trace operation, and $\operatorname{diag}\left(a_{1}, a_{2}, \ldots, a_{n}\right)$ refers to the square matrix with diagonal elements given by $a_{1}, a_{2}, \ldots, a_{n}$. The cardinality of the set $\mathcal{K}$ is expressed as $|\mathcal{K}| \operatorname{det}(\mathbf{A})$ is the determinant of $\mathbf{A}, \operatorname{abs}(a)$ is the absolute value of $a$, $a^{+}=\max (a, 0)$, and $\operatorname{tr}(\mathbf{A})^{-1}$ is the trace of $\mathbf{A}^{-1}$. The
$Q \times Q$ identity matrix is $\mathbf{I}_{Q}$, and the vector $\mathbf{1}_{k}$ has zeros at all positions except the $k$ th.

We focus on the downlink, where a $Q$-antenna AP communicates simultaneously with $K$ single-antenna terminals, under the assumption that $K \leq Q$. Certainly, in a practical situation $K>Q$, thus some kind of user grouping should be performed, see e.g. Chapter 5 in [2] or [10]. The analysis presented here is valid for each group of users that would be formed in those cases. At any time instant, the signal model is expressed as

$$
\mathbf{y}=\mathbf{H B s}+\mathbf{w} \in \mathbb{C}^{K \times 1},
$$

where the $k$ th position of vector $\mathbf{y}(\mathbf{s})$ is the received (transmitted) signal for user $k$. $\mathbf{H}$ is the $K \times Q$ complex flat-fading channel matrix, whose $i$ th row contains the $1 \times Q$ vector of the channel gains for the $i$ th user, i.e. $\mathbf{h}_{i}^{T}$. The channel matrix elements are independent and identically distributed complex Gaussian random variables with zero mean and unit variance, and it is assumed to be perfectly known at the transmitter. The noise vector is complex Gaussian, i.e. $\mathbf{w} \sim \mathcal{N}_{\mathbb{C}}\left(\mathbf{0}, \sigma^{2} \mathbf{I}_{K}\right)$. The transmit beamvectors are gathered in the matrix $\mathbf{B}=$ $\left[\mathbf{b}_{1} \mathbf{b}_{2} \ldots \mathbf{b}_{K}\right] \in \mathbb{C}^{Q \times K}$. Assuming that the transmitted signal $\mathbf{s}$ has unitary mean energy and the power budget is $P_{T}$, then $\operatorname{tr}\left(\mathbf{B}^{H} \mathbf{B}\right) \leq P_{T}$ should be fulfilled instantaneously. The active terminals (users) are gathered in the set $\mathcal{K}=\{1, \ldots, K\}$. Note that this signal model can be seen as a subcarrier of an Orthogonal Frequency Division Multiplexing (OFDM) system.

$\mathrm{ZF}$ is a linear processing technique, which is equivalent to the MMSE for high SNR [11]. Moreover, it yields a reasonable degradation with respect to the optimum sum capacity as it is shown in [12]. ZF implies that the $K$ users see parallel and orthogonal fading channels corrupted only by Additive White Gaussian Noise and not by interference signals from other users. Particularly, the interference signals in (1) can be completely eliminated by creating parallel and orthogonal spatial channels, thus the signal received for the $k$ th user is only corrupted by noise. The equivalent channel is captured by $\alpha_{k}$, so that the beamforming criterion becomes $\mathbf{H} \widetilde{\mathbf{B}}=\mathbf{D}_{\alpha}$, where $\mathbf{D}_{\alpha}=\operatorname{diag}\left(\alpha_{1}, \alpha_{2}, \ldots, \alpha_{K}\right)$ [13]. Since

$$
\alpha_{k}^{2}=1 /\left[\left(\mathbf{H H}^{H}\right)^{-1}\right]_{k, k},
$$

it is essential that the channel covariance matrix is wellconditioned, i.e. the channels from the users should not arrive from close directions in the spatial domain. The $\alpha_{k}^{2}$ behave like independent central Chi-Square random variables with $2(Q-K+1)$ degrees of freedom, i.e. $\alpha_{k}^{2} \sim \frac{1}{2} \chi_{2(Q-K+1)}^{2}$ [2]. However, they concentrate the effect of the choice of the users that are simultaneously served, because they depend on the inverse of the matrix $\mathbf{H} \mathbf{H}^{H}$. If the channel vectors are highly correlated, then the determinant of the matrix $\mathbf{H} \mathbf{H}^{H}$ tends to 0 , and consequently, the equivalent spatial channels in (2) tend also to null out. As an extreme example, if two rows were exactly the same, the previous matrix would be ill-conditioned and the inverse would not exist. Relaxing this, if the rows of the matrix $\mathbf{H}$ are highly correlated, i.e. the channels refer to a close spatial zone, more power is needed to fulfill the requirements of any user.

As it has been stated, the beamforming matrix contains the power allocation, i.e. $\mathbf{B}=\widetilde{\mathbf{B}} \mathbf{D}_{\beta}$, where $\mathbf{D}_{\alpha}=$ 
$\operatorname{diag}\left(\beta_{1}, \beta_{2}, \ldots, \beta_{K}\right)$, so the signal model in (1) finally reduces to

$$
\mathbf{y}=\mathbf{D}_{\alpha} \mathbf{D}_{\beta} \mathbf{s}+\mathbf{w} \Rightarrow y_{k}=\alpha_{k} \beta_{k} s_{k}+w_{k},
$$

so the SNR for the $k$ th user is

$$
\gamma_{k}=\frac{\alpha_{k}^{2} \beta_{k}^{2}}{\sigma^{2}}
$$

where it has been assumed that the symbols have unitary mean energy, particularly, normalized QAM symbols, and it is considered without loss of generality that the noise power $\sigma^{2}$ is equal for the $K$ users. Since simplicity is an important feature for schedulers, it is necessary to use an easydifferentiable BER expression. Therefore, it is meaningful to use the approximate BER for QAM signals in Rayleigh fading channels corrupted only by AWGN given in [14], i.e.

$$
\operatorname{BER}(\gamma) \approx c_{1} \exp \left(-c_{2} \gamma\right)
$$

where $c_{1}=0.2, c_{2}=\frac{1.6}{2^{m}-1}$, and $m$ is the number of bits in the constellation, fixed for the purpose of this paper. This expression is valid within $1.5 \mathrm{~dB}$ of error for a BER $\leq 10^{-3}$. If several signal mappings are available, the throughput (rate) depends on the number of bits of the symbols and on the BER. At the physical layer, rate is the maximum number of bits per symbol $m$ that can be transmitted while fulfilling a

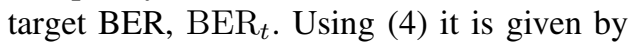

$$
m=\log _{2}\left(1+\frac{\gamma}{\Gamma}\right),
$$

where the constant $\Gamma$ is given by $\Gamma=\frac{\log \left(c_{1} / \mathrm{BER}_{t}\right)}{c_{2}}$. In fact, $\Gamma=1$ can be interpreted as the classical Shannon's limit to error-free bit rate (capacity) [15]. Typically, $m$ is a real number, and an spatial waterfilling can be performed in order to achieve the maximum sum rate of the SDMA channel, as it is shown in the next section. Other schemes may provide a lower sum rate but ensure a more fair resource distribution. In practical systems only a finite set of mappings is usually available, thus $m$ is an integer, see [10] for details.

\section{Power Allocation Techniques}

In this section, we propose several alternatives for allocating the total available power $P_{T}$ to the users. We assume that they are homogeneous, i.e. their requirements are the same in terms of delay, throughput, and BER. Given a number of users, the BS tries to obtain the best resource sharing. Nevertheless, the BS does not optimize the number of users nor selects the best users to serve (see Section V for more details). Besides, we assume the use of a single symbol mapping. In the downlink, cost functions usually aim at optimizing the BER or rate while constraining the power, although other meaningful options minimize the total power subject to a BER or rate constraint [16]. In this paper, we focus on the first possibility for the sake of conciseness.

\section{A. Uniform Power Allocation (UPA)}

Without any channel knowledge, the best option reduces to the well-known UPA. In that case, the AP divides equally the whole power among the active users in the cell, so that it does not care about their actual channel gain nor how the performance might be improved. Basically,

$$
\beta_{k}^{2}=\frac{P_{T}}{K}
$$

is the power allocated to the $k$ th user, so the SNR for the $k$ th user is given by

$$
\gamma_{k}=\frac{P_{T}}{\sigma^{2}} \frac{\alpha_{k}^{2}}{K}
$$

which leads to a higher rate (and lower BER) for the users having a better channel. Since it is assumed that the BS has perfect channel knowledge, a more efficient power allocation criterion might be applied. In fact, the fairness criterion determines the power allocation. A first option assigns the same amount of resource to the users, which is translated into the same BER and rate. However, other fairness considerations state that the users with a higher mean SNR during a certain time window shall be provided a higher rate than those having a poorer link quality ${ }^{2}$. Then, a second option optimizes the global performance regardless of the users with worse channel conditions, including the maximum sum rate technique and the strategy minimizing the sum BER that are presented next. This SDMA scheme offers a significant performance improvement over opportunistic communications such as [17], since several users share the spatial channel and not only the one with a proportionally better channel [2]. A last remark is that the techniques that are presented can be considered as a besteffort type of service, since the BS optimizes the BER or the rate regardless of the individual QoS achieved by the active users. For the fulfillment of individual requirements, please see next section.

\section{B. Equal Rate and BER Scheme (ERB)}

A possible optimization criterion consists of assigning the same rate and BER to all users, regardless of their channel quality, i.e. we would like to maximize the minimum user rate

$$
\begin{aligned}
& \max _{\beta_{k}^{2}} \min _{k} m_{k} \\
& \text { s.t. } \sum_{k \in \mathcal{K}} \beta_{k}^{2} \leq P_{T},
\end{aligned}
$$

which implies that the product $\alpha_{k} \beta_{k}$ is the same for all users and equal to a constant $\bar{\beta}$. Note that this strategy can also be expressed as the minimization of the maximum BER. Applying the constraint on the total power, $\bar{\beta}$ reduces to

$$
\bar{\beta}=\sqrt{\frac{P_{T}}{\operatorname{tr}\left(\mathbf{H H}^{H}\right)^{-1}}},
$$

so the SNR is the same for the $K$ users, i.e. $\gamma_{k}=\gamma, \forall k \in \mathcal{K}$, and it is given by

$$
\gamma=\frac{P_{T}}{\sigma^{2}} \frac{1}{\operatorname{tr}\left(\mathbf{H H}^{H}\right)^{-1}} .
$$

With this technique, a high amount of power is used for the users that have a poor channel quality, which degrades the

\footnotetext{
${ }^{2}$ This might also be applied if the price of the service varies depending on the desired QoS.
} 
TABLE I

SPATIAL WATERFILLING ALgORithm

1. Set $\mathcal{K}=\{1, \ldots, K\}$.

2. Build matrix $\mathbf{H}$ for the users in the set $\mathcal{K}$.

3. Compute $\alpha_{k}^{2}=1 /\left[\left(\mathbf{H H}^{H}\right)^{-1}\right]_{k_{k}, k}, \forall k \in \mathcal{K}$.

4. Compute $\mu^{-1}=\frac{P_{T}+\sigma^{2} \sum_{k \in \mathcal{K}} \alpha_{k}^{-2}{ }^{2}}{|\mathcal{K}|}$.

5. Compute the power allocation factors as in (13).

6. If $\beta_{k}^{2}>0, \forall k \in \mathcal{K}$, then the algorithm finishes. Else, remove the users having zero power $\mathcal{K} \leftarrow \mathcal{K}-$ $\left\{k \in \mathcal{K}: \beta_{k}^{2}=0\right\}$, and go to step 2 .

performance of the better users, thus the global performance of the cell is penalized. As stated in Appendix I, the ERB achieves always a lower sum rate than the UPA, but it has the powerful property that all the users are granted the same service. Indeed, it penalizes the global performance for the sake of the individual revenues.

\section{Maximum Sum Rate (MSR)}

Instead of guaranteeing the same SNR for all users, thus also BER and rate, another option is to maximize the sum rate of the cell, without considering a possibly uneven resource partitioning. Some users might not be scheduled, allowing others to have a higher rate and a lower BER. It is important to note that since perfect information is available at the AP, not only the best user will be scheduled for transmission, but rather a subset of all the terminals that are active. The AP penalizes the users with poorer channels, and increase the performance of the better users, thus the global performance of the cell. This cost function is expressed as

$$
\begin{aligned}
& \max _{\beta_{k}^{2}} \sum_{k \in \mathcal{K}} m_{k} \\
& \text { s.t. } \sum_{k \in \mathcal{K}} \beta_{k}^{2} \leq P_{T},
\end{aligned}
$$

where $m_{k}$ is a real number reflecting the maximum achievable rate for the $k$ th user, recall (5). After the application of the KKT conditions to this problem [18], the following modified water-filling algorithm is obtained:

$$
\beta_{k}^{2}=\left(\mu^{-1}-\frac{\sigma^{2}}{\alpha_{k}^{2}}\right)^{+},
$$

where $\mu$ is chosen to satisfy the power constraint in (12) with equality. The SNR for the $k$ th user is then given by

$$
\gamma_{k}=\left[\left(\mu^{-1}-\frac{\sigma^{2}}{\alpha_{k}^{2}}\right) \frac{\alpha_{k}^{2}}{\sigma^{2}}\right]^{+} .
$$

It is proven in Appendix II that the UPA tends to the MSR in the high SNR regime. The implementation is detailed in Table I. First, the scheduler tries to serve all the users, steps 1 and 2. It calculates the equivalent channels after the beamforming $\alpha_{k}$ (step 3 ) and computes the water level for that configuration (step 4). With it, it obtains the power allocation factors in step 5. If some users cannot reach the water level, they are removed from the active set (step 6). At this point, it is essential that the equivalent channels $\alpha_{k}^{2}$ are recomputed (step
2) since they depend on the users that are being served. This procedure is repeated until all the users in the set of active users are assigned a non-zero power (finishing condition in step 6).

\section{Minimum Sum BER (MSB)}

Instead of rate methods, another possibility is to minimize the total BER. In fact, with a single constellation, a lower BER implies a higher throughput. We would like to minimize the sum BER of all the users in the cell subject to the power constraint, i.e.

$$
\begin{aligned}
& \min _{\beta_{k}^{2}} \sum_{k \in \mathcal{K}} \mathrm{BER}_{k} \\
& \text { s.t. } \sum_{k \in \mathcal{K}} \beta_{k}^{2} \leq P_{T} .
\end{aligned}
$$

Using (4) and taking derivatives of the Lagrangian of the problem with respect to the power allocation factors $\beta_{k}^{2}$ and to the multiplier $\mu$, the solution for the power factors is

$$
\beta_{k}^{2}=\frac{\sigma^{2}}{c_{2} \alpha_{k}^{2}}\left(\log \left(c_{1} c_{2} \frac{\alpha_{k}^{2}}{\sigma^{2}}\right)-\log (\mu)\right)^{+},
$$

where $\mu$ is obtained by imposing the constraint in (15). Similarly to the MSR, some users might not transmit, thus the packets in their queues will be either lost or deferred depending on the delay constraints of the application. The performance of the MSB in terms of BER tends to that of the ERB at high SNR, as we prove in Appendix III. A last remark is that it can be stated that an upper bound of the sum BER is minimized by the UPA if we have no knowledge about the channel or if the quality of the estimation is very low [2]. Since this scheme can be seen again as a modified waterfilling, it is implemented in a similar way as the algorithm in Table I. Therefore, we do not detail the algorithmic implementation.

\section{E. Simulation results}

Up to this point, several traditional power allocation techniques have been presented, and it has been theoretically shown in the appendices that asymptotically in the high SNR regime,

1) the sum rate of the ERB is always lower than that of the UPA;

2) the sum rate of the MSR tends to be the same as that of the UPA;

3) the performance in terms of BER of the BER-based techniques, namely the MSB and the ERB, tends to be the same.

In this section, these results will be shown through simulations, as well as a new perspective to show the fairness of the proposed power allocation techniques will be given, namely the mean vs. standard deviation plots for each selected metric [2]. Indeed, it is important to see graphically the behavior of the proposed strategies and not only in global performance as it is done usually at the physical layer. An AP provided with $Q=6$ antennas is the transmitter, and the SNR in the figures refers to the ratio $\frac{P_{T}}{\sigma^{2}}$. In order to compare the power allocation strategies, the number of users is equal to 


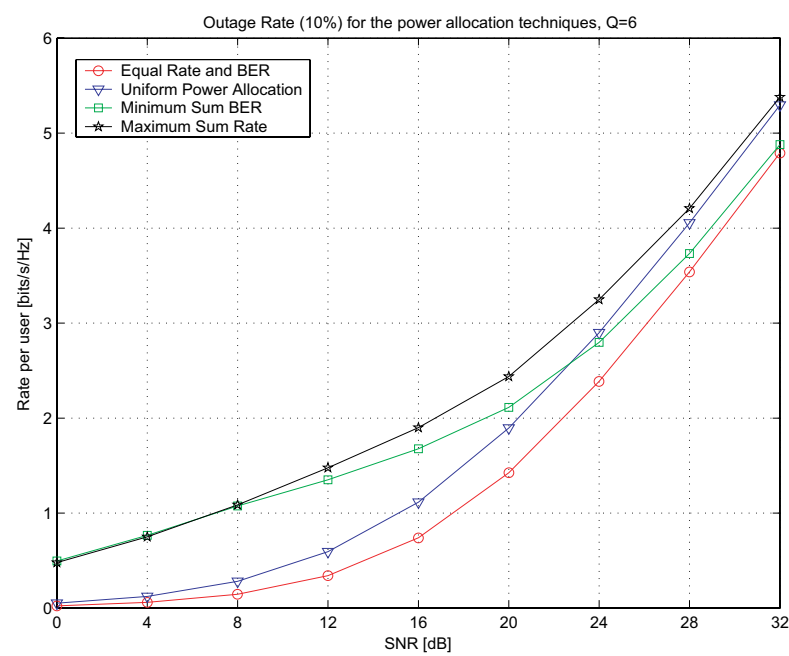

Fig. 2. For the proposed power allocation techniques, the figure shows the outage mean rate at $10 \%$ vs. SNR. The equivalences at high and low SNR are clear.

the number of antennas, i.e. $K=Q$, and the gap in (5) is set to $\Gamma=1$ because these methods do not take into account the BER constraints for the moment. The signal mapping that has been assumed is QPSK without loss of generality.

In Fig. 2, one finds the outage rate at $10 \%$ vs. the SNR. With an outage rate of $R$ at $\mathrm{x} \%$ it is meant that $\mathrm{x} \%$ of the time the rate is below $R$, or equivalently, that a minimum rate of $R$ is guaranteed $100-\mathrm{x} \%$ of the time. Conversely for the BER, the outage is usually $90 \%$, which means that the BER is $90 \%$ of the time below the plotted value. On the other hand, Fig. 3 compares the proposed techniques in terms of BER vs. the SNR. Several observations can be made from the two figures. As stated, the ERB and the MSB tend to achieve the same performance both in terms of BER and rate in the high SNR regime, whereas the UPA and the MSR tend to obtain the same average performance at high SNR. As expected, the rate is maximized by the MSR, whereas the BER is minimized by the MSB. Another interesting comparison is at the low SNR regime. There, the ERB and the UPA achieve very similar performance both in terms of BER and rate. On the other hand, the MSR and the MSB are equivalent at low SNR, since it is in that region where the number of served users is very low, typically at low SNR only the best user shall be scheduled for transmission. As a final remark, it should be noted that if bit allocation strategies were used in this case, the BER methods would always choose robust constellations because they obtain the lowest BER, whereas the methods based on rate tend to choose the constellations with a higher number of bits since the highest rate is then achieved. For further details on bit allocation strategies, please refer to [2]. The figures that have been shown up to now are traditional in the literature, however, they do not reflect how the resources are distributed among the users, which is a key part of the dissertation.

Among other options in [2], the preferred plot to show fairness at this point is the mean vs. an approximation of the standard deviation for each of the selected metrics (rate and BER). As it will be stated, compared to traditional asymptotic analysis in the literature, this plot provides information not only at high or low SNR, but also at the intermediate points,

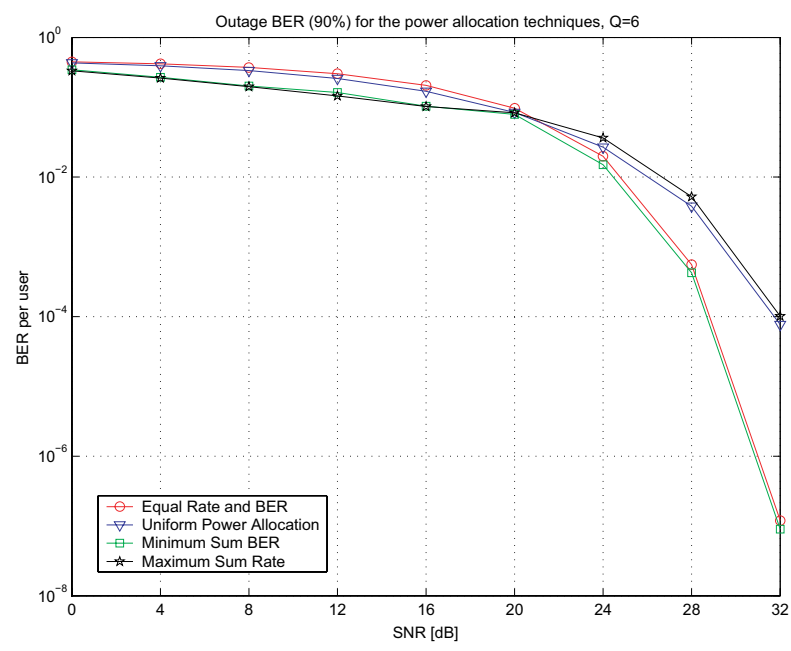

Fig. 3. For the proposed power allocation techniques, the figure shows the outage mean BER at $90 \%$ vs. SNR. The equivalences at high and low SNR are clear.

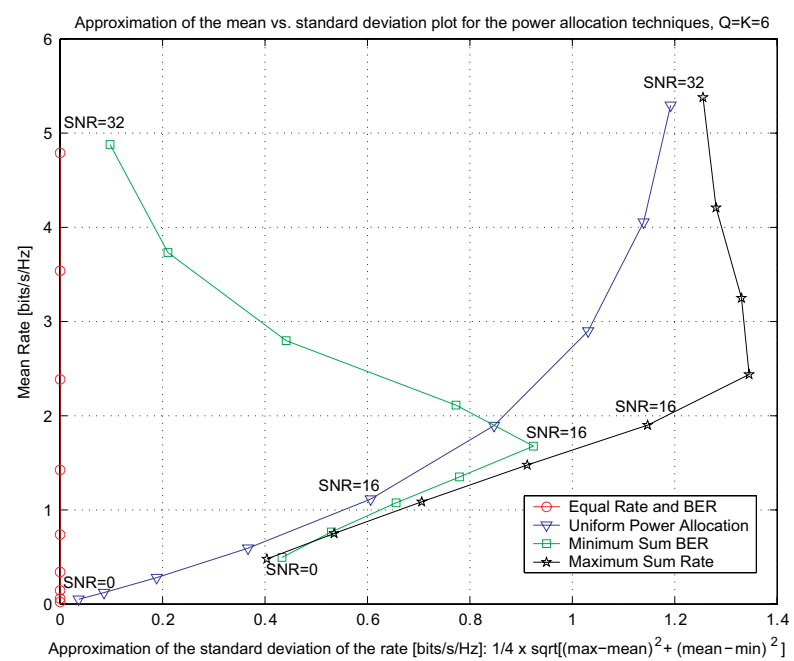

Fig. 4. For the proposed power allocation techniques, the figure shows the outage mean rate vs. the approximation of the standard deviation at $90 \%$ SNR.

which are usually the operating points of realistic systems. First, Fig. 4 shows the mean rate vs. the standard deviation, and Fig. 5 is devoted to the BER. It shall be noted here that each point in the figures refers to a SNR value, ranging from $0 \mathrm{~dB}$ to $32 \mathrm{~dB}$ in steps of $4 \mathrm{~dB}$, so there is a total of 9 points per method. Moreover, the ERB curve is denoted by circles, the MSB by squares, the MSR by stars, and the UPA by triangles. In both Fig. 4 and Fig. 5, it can be stated that the ERB provides an equal performance for all the users, since it is a line at the coordinate axis. The equivalences in terms of rate at high are also clear: the MSB tends to the ERB at high SNR, and the UPA converges in rate to the MSR. An interesting performance plot is that of the MSB, since at low SNR it tends to the MSR, whereas at high SNR it approaches the rate of the ERB.

The behavior of the techniques shown in these plots has been already identified in the previous comparisons, but it is rather interesting to reflect it in a figure. Regarding the BER plot in Fig. 5, the results are not so clear as for the 


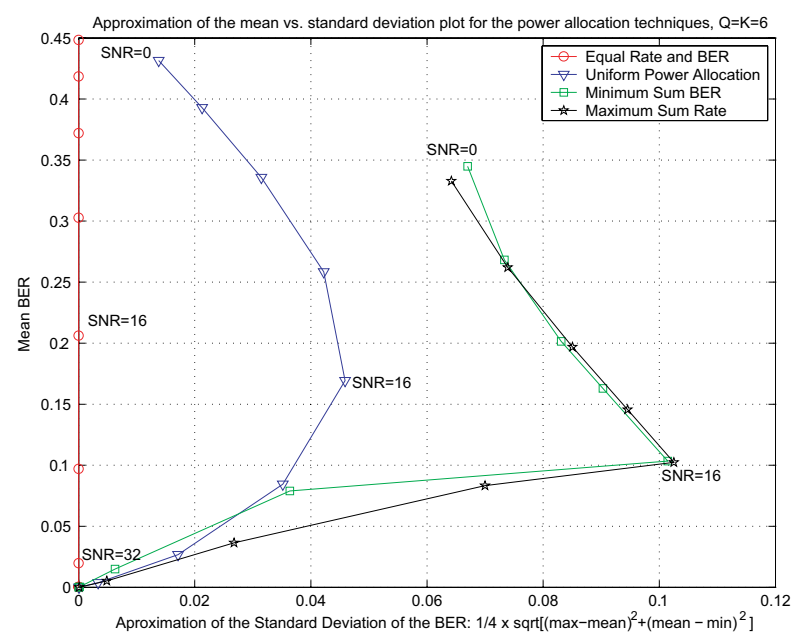

Fig. 5. For the proposed power allocation techniques, the figure shows the outage mean BER vs. the approximation of the standard deviation at $90 \%$ SNR.

rate metric, although the same comments as before shall be made. However, one does not distinguish so well the behavior of the MSB. Finally, the methods that have a better global performance tend to distribute the resources in an uneven way in both figures, see e.g. the MSR in Fig. 4. For each SNR, the mean performance is the best, but the variance among the users is also higher than for the other methods. Last, one can observe that the MSR and the MSB have a sharp change in their trend in Fig. 4 and Fig. 5, which is due to the nature of their waterfilling-like solution: when there is low SNR they tend to serve a low number of users, whereas up from a certain point, the power can be shared among all the users.

\section{THE FAIR BALANCE: \\ THE EQUAL PROPORTIONAL SNR (EPS)}

From the previous results, the scheduler might select the ERB and the UPA for simplicity, as well as to deal with other mechanisms such as the admission control. However, before going into deeper details on these issues, a new method providing an intermediate performance among them shall be described, the EPS. In fact, the EPS is both a power allocation and an admission control mechanism, see Section V. A first presentation and comparison with respect to the UPA and the ERB is needed in order to show the benefits of such a technique in terms of fairness. The EPS is based on the fact that the users might agree to loose the same proportion $\delta_{k}$ of their maximum achievable SNR, $\gamma_{k}^{a}$, which is obtained as if they were served alone in the cell, i.e.

$$
\gamma_{k}^{a}=\gamma^{n}\left\|\mathbf{h}_{k}\right\|^{2}
$$

where the channel $\mathbf{h}_{k}$ is the $k$ th row of the complete matrix H. In fact, $\delta_{k}$ can be seen as the price paid for the collective satisfaction and could be computed according to the traffic requirements. If the terminals belong to the same network, e.g. at home, this might be a criterion to determine the access to the core, and it can be classified into a new metric for fairness. Mathematically, the fraction of the maximum achievable SNR is given by

$$
\delta_{k}=\frac{\gamma_{k}}{\gamma_{k}^{a}}, \forall k \in \mathcal{K}
$$

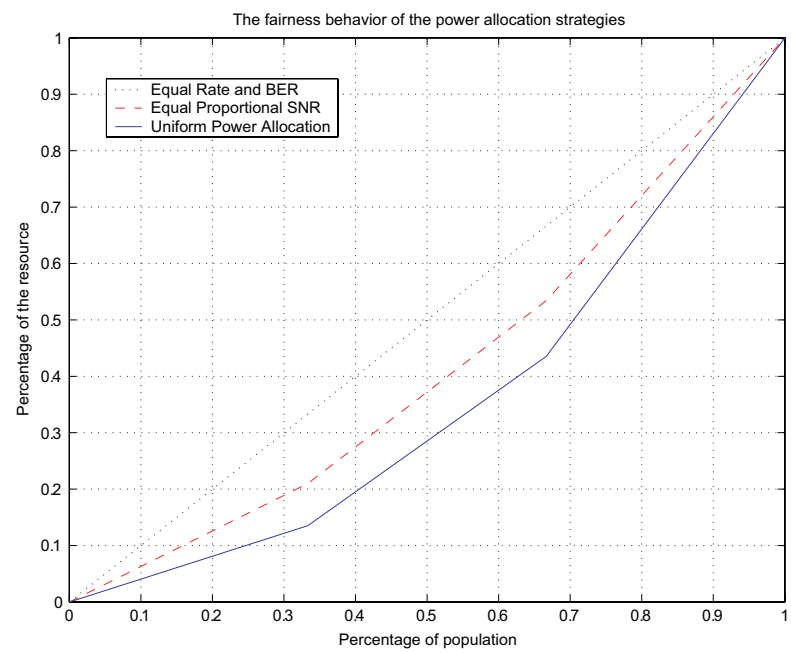

Fig. 6. For $Q=20$ antennas and $K=16$ users, the fairness curves for the UPA (solid), the ERB (dotted), and the EPS (dashed).

If all the users are homogeneous and allow the same loss in proportion to their maximum SNR, i.e. $\delta_{k}=\delta, \forall k \in \mathcal{K}$, the cost function of this problem is expressed as

$$
\begin{aligned}
& \max \delta \\
& \text { s.t. } \\
& \sum_{k \in \mathcal{K}} \beta_{k}^{2} \leq P_{T},
\end{aligned}
$$

which has the nice property of yielding a closed-form solution for $\delta$,

$$
\delta^{-1}=\sum_{k \in \mathcal{K}} \frac{\left\|\mathbf{h}_{k}\right\|^{2}}{\alpha_{k}^{2}},
$$

but ultimately attains a different SNR for each user given by

$$
\gamma_{k}^{E P S}=\delta \gamma_{k}^{a}=\gamma^{n} \delta\left\|\mathbf{h}_{k}\right\|^{2}=\gamma^{n} \frac{\left\|\mathbf{h}_{k}\right\|^{2}}{\delta^{-1}}
$$

The suitability of this strategy is shown next by means of an approximation of Gini plot for the UPA, the ERB, and the newly proposed EPS. Details about the analysis can be found in Appendix IV, in which the analysis of the mean SNR as well as the maximum and minimum SNR for the users is given for the proposed schemes. It is shown in Fig. 6 that the ERB yields an equal distribution of the resource, i.e. for a given percentage of the population ( $\mathrm{x}$ axis), the same fraction of the resource (y axis) is obtained. Increasing the distance with respect to this curve also increases the differences among users, because in that case the slope is small for low percentage of the population, whereas the slope starts to increase when the percentage of the population becomes higher. Then, the most unfair solution is the UPA (solid line) because it yields the higher area between its line and that of the ERB (dotted curve). Between the UPA and the ERB one finds the EPS (dashed line), which achieves not only an intermediate mean value, but also average maximum and minimum values. To conclude, a good balance between the global performance and the individual needs is obtained with the EPS.

\section{Spatial Admission Control}

In fact, some kind of admission control or user selection is already being made in the previous algorithms whenever a 


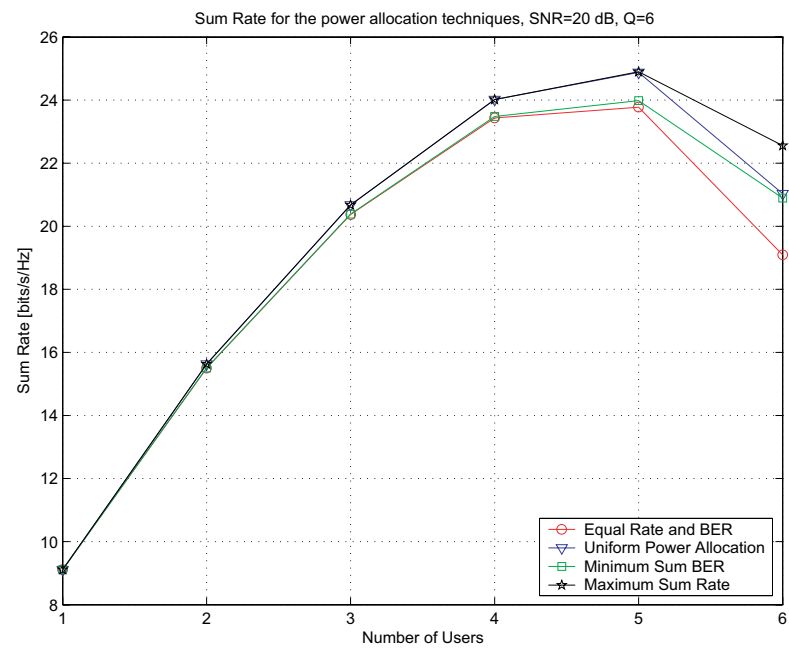

Fig. 7. For $Q=6$ antennas, mean sum rate vs. number of users for the power allocation techniques: the ERB, the UPA, the MSB, and the MSR.

user shall be removed from the active set because the power constraint cannot be fulfilled. However, the previous strategies for the power allocation obtain the solution for a given number of antennas $Q$ and users $K$, and the BS does not control the individual QoS for the users in a best-effort type of service. In the SDMA system that has been proposed, a maximum number of $Q$ users can be allocated for transmission, that is, one antenna, one user, but the optimum number of users might be lower than $Q$. This fact leads the BS to allocate the best users in order to obtain the best performance. The optimization at the BS shall determine both the number of users and, more concretely, which users, since the interactions among them are crucial for the performance of the scheduler [2].

Linked to the choice of the optimum number of users, it has been recently reported that there exists an optimum number of antennas that should be dedicated to the users if $\mathrm{ZF}$ is used as transmit beamforming scheme [19]. Equivalently, the best global performance is achieved when less than $Q$ users are served simultaneously. If the number of antennas $Q$ is higher than the number of active users $K$, and they both grow without bound, i.e. $K, Q \rightarrow \infty$, but their ratio $\zeta=\frac{Q}{K}$ remains constant, the sum rate $R$ increases linearly with respect to the number of antennas not only for the ERB technique [19], but also for the UPA and the MSR, i.e.

$$
\lim _{K, Q \rightarrow \infty} \frac{R}{Q}=\frac{1}{\zeta} \log \left(1+\frac{P_{T}}{\sigma^{2}}(\zeta-1)\right) .
$$

Looking at the previous equation, one should note that there exists a number of users $K$ that optimizes the sum rate for any given number of antennas $Q$. If the number of antennas $Q$ is fixed, the BS has to select the optimum number of users $K$. Besides, the sum rate can differ significantly depending on the choice of these $K$ users, since if their channels are correlated, then more power is needed. Fig. 7 depicts the mean sum rate vs. the number of users being simultaneously served at the array, for the ERB, the UPA, the MSB, and the MSR. In any case, the sum rate is maximized if the number of users is lower than the number of antennas. In fact, serving as many users as antennas penalizes the performance. However, note that if one looks at the rate per user, with 5 users, the rate for

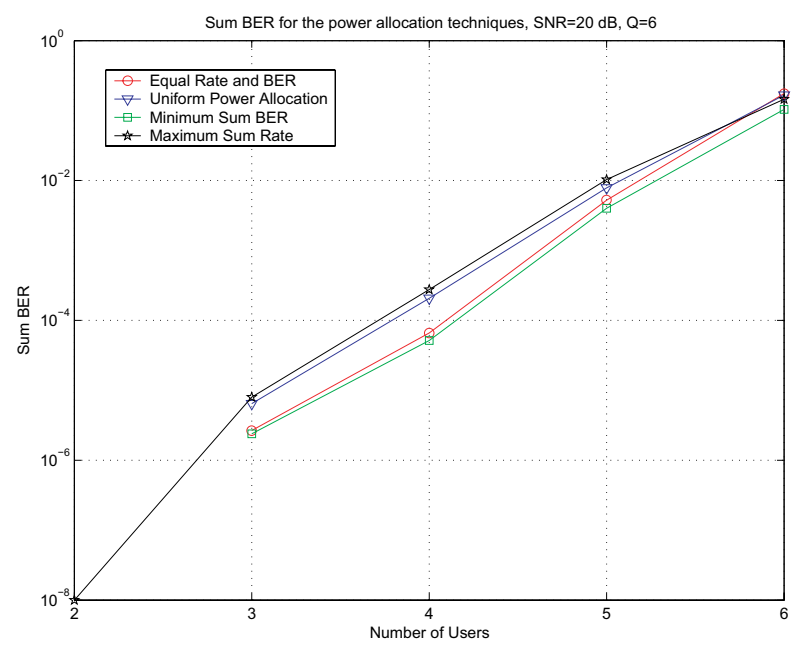

Fig. 8. For $Q=6$ antennas, mean sum BER vs. number of users for the power allocation techniques: the ERB, the UPA, the MSB, and the MSR.

the MSR is about 5 bits/s/Hz, whereas for a single user, this value is nearly doubled. Therefore, the performance decreases in terms of rate per user although the sum rate might be better. Differently to the rate methods, for the BER techniques the sum BER always decreases as the number of users decreases. It is shown in Fig. 8 the mean BER performance vs. the SNR for the ERB, the UPA, the MSB, and the MSR with 4-QAM (QPSK) mapping. The BER can be dramatically reduced if the scheduler serves two users less than the number of antennas.

This balance between the BER and the rate is important in practical scenarios, where the BS aims at optimizing the global performance of the cell while trying to cope with the individual QoS requirements of the users. For this purpose, the Spatial Admission Control (SAC) mechanism decides which users cannot be scheduled while fulfilling the requirements of the selected ones and optimizing the global performance at the same time. Due to the asymptotic behavior discussed previously in this paper, only the UPA and the ERB technique are taken as benchmarks for a comparison with the newly proposed EPS. It has been shown in [2] that the diversity order of the UPA is always higher than that of the ERB, and that the EPS provides an intermediate performance among them. In this section, it will be shown that the number of served users (multiplexing gain) is higher for the ERB than for the UPA, and the EPS also provides an intermediate behavior.

\section{A. The addition of SNR constraints}

The main goal of the PHY scheduler is to reduce the amount of information that shall be processed by the traffic scheduler at the DLC. Particularly, the PHY scheduler performs the admission control. Due to the interactions in this SDMA system, a crucial point is which subset of users $\mathcal{K}$ is served. This shall be decided taking into account the BER or rate requirements, which can be mapped into a target SNR $\gamma^{t}$. The feasibility conditions for the UPA, the ERB, and the EPS with SNR constraints are provided, after which some simulation results are given. 
TABLE II

SPATIAL ADMISSION CONTROL.

1. Set $\mathcal{K}=\{1, \ldots, K\}$.

2. Build matrix $\mathbf{H}$ for the users in the set $\mathcal{K}$.

3. Compute $\alpha_{k}^{2}=1 /\left[\left(\mathbf{H H}^{H}\right)^{-1}\right]_{k, k}, \forall k \in \mathcal{K}$.

4. If the condition (depending on the technique) in Section V-A.1 is satisfied, go to step 7 .

5. Otherwise, remove the active user having the worst channel $\mathcal{K} \leftarrow \mathcal{K}-\left\{k^{*} \in \mathcal{K}: \min _{k} \alpha_{k}^{2}\right\}$, and go to step 2.

6. If $|\mathcal{K}|=\emptyset$, the algorithm finishes.

7. Compute the power allocation according to one of the criteria in Section V-A.1, and finish.

1) Feasibility conditions: First, for the UPA, the SNR for each user $k$ shall be above the threshold $\gamma^{t}$, i.e.

$$
\gamma_{k}^{U P A}=\gamma^{n} \frac{\alpha_{k}^{2}}{K} \geq \gamma^{t}
$$

so that the equivalent channel for each user shall fulfill

$$
\alpha_{k}^{2} \geq|\mathcal{K}| \frac{\gamma^{t}}{\gamma^{n}}, \forall k \in \mathcal{K}
$$

where it is emphasized that the set of users $\mathcal{K}$ that shall be served is optimized. On the other hand, for the ERB

$$
\operatorname{tr}\left[\left(\mathbf{H H}^{H}\right)^{-1}\right] \leq \frac{\gamma^{n}}{\gamma^{t}}
$$

shall be fulfilled, which is a single constraint for all the users although it requires the same computational complexity. Finally, for the EPS the constraints are again individual, so that

$$
\left\|\mathbf{h}_{k}\right\|^{2} \geq \delta^{-1} \frac{\gamma^{t}}{\gamma^{n}}, \forall k \in \mathcal{K},
$$

shall be fulfilled.

The admission control mechanism is summarized in Table II. It tries to fit all the users (steps 1 to 4 ), but if the feasibility condition for the selected technique is not satisfied (step 4) the user with the lowest equivalent channel is removed from the active set $\mathcal{K}$ (step 5), since it is the one that worsens the performance of the other users, as stated by the solvability conditions. When the solvability condition is fulfilled, the power allocation factors are computed, see step 7. On the other hand, if the condition cannot be fulfilled with any user, the algorithm finishes and does not serve any user (step 6). Since the AP starts from the maximum number of users and then drops the worst user out at each iteration, the optimum distribution of the users might be found.

In order to evaluate the performance of this spatial admission control mechanism, the following setup is built. The cell is governed by an $\mathrm{AP}$ with $Q=8$ antennas, and there is a maximum of $K=8$ users in the cell. However, since the SNR constraints shall be fulfilled, not all of them will be served. It is assumed that the SNR is $\gamma^{n}=\frac{P_{T}}{\sigma^{2}}=30 \mathrm{~dB}$, so that the system operates in the high SNR regime, and the target SNR is varied from 5 to $30 \mathrm{~dB}$. Fig. 9 reflects the results that have been

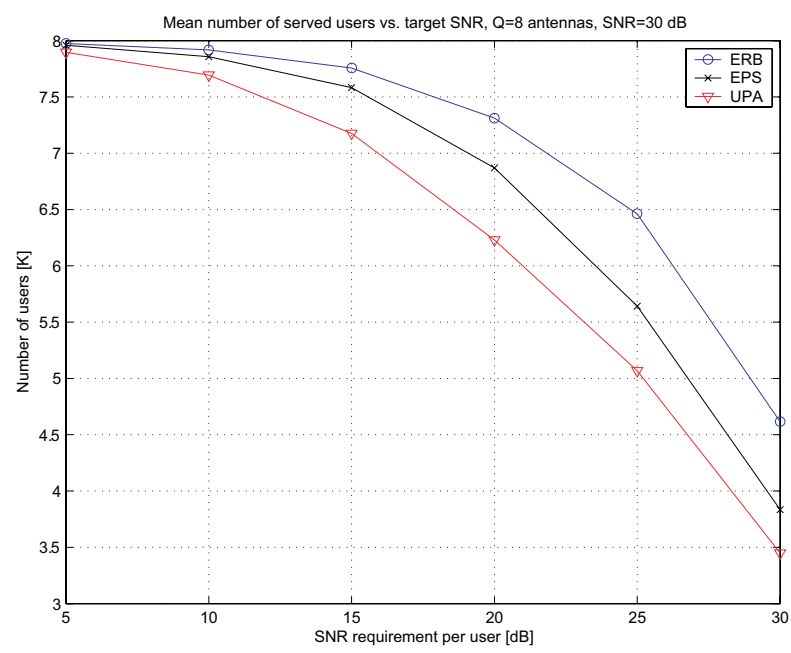

Fig. 9. For $Q=8$ antennas, mean number of served users vs. the SNR requirement.

previously exposed from another perspective, namely the mean number of served users with respect to the SNR requirement, which reflects in some sense the multiplexing gain. It is stated that the UPA gives service to the lowest number of users so as to improve the global performance by not serving the poorer users. On the other hand, the ERB serves the highest number of users, but the global performance is penalized, as it has been seen before. Finally, the EPS strategy provides again an intermediate solution between them. Therefore, recalling the results concerning the diversity gain, it can be stated that more diversity gain implies a lower multiplexing gain, which agrees with the trade-off in [20].

\section{Vi. Conclusions}

This paper is devoted to the power allocation techniques in a multi-antenna broadcast channel. First, several traditional criteria have been formulated and analyzed, especially in terms of fairness, which is an issue that is usually forgotten in the physical layer literature. Depending on the optimization goal of the PHY-DLC scheduler, several options are available to balance the individual needs and the cell needs. Moreover, their correspondences in the high SNR regime have been evaluated, so that finally the Uniform Power Allocation (UPA) and the scheme providing an equal SNR (thus rate and BER) for every user (ERB) are the selected techniques for the admission control. They are asymptotically equivalent to their counterparts: the Maximum Sum Rate (MSR) and the Minimum Sum BER (MSB). After that, the ERB and the UPA are compared to a new strategy, the Equal Proportional SNR (EPS), which balances in an intermediate way the tradeoff among the global optimization and the fulfillment of the individual constraints. Moreover, the admission control procedure reflects the fundamental trade-off between the diversity gain and the multiplexing gain for these power allocation techniques. The EPS is shown to provide an intermediate behavior among the UPA and ERB.

\section{ACKNOWLEDGEMENTS}

The authors want to thank the Editor, Prof. Li-Chun Wang, and the anonymous reviewers for their valuable comments. 


\section{APPENDIX I \\ THE SUM RATE OF THE ERB IS \\ ALWAYS LOWER THAN THAT OF THE UPA}

Taking the approximation of a high SNR, it can be easily proven that the ERB achieves always a lower sum rate than the UPA. In the high SNR regime, the sum rate of the ERB $R_{T}^{E R B}$ can be expressed as

$$
\begin{aligned}
R_{T}^{E R B} & \approx K \log \left(\frac{P_{T}}{\sigma^{2}} \frac{1}{\operatorname{tr}\left(\mathbf{H H}^{H}\right)^{-1}}\right)= \\
& =K \log \left(\frac{P_{T}}{\sigma^{2}} \frac{1}{\sum_{k \in \mathcal{K}} \frac{1}{\alpha_{k}^{2}}}\right)=C T+K \log H_{\alpha}
\end{aligned}
$$

where $H_{\alpha}$ is the harmonic mean of the $\alpha_{k}^{2}$ and $C T$ is a constant, i.e.

$$
\begin{aligned}
\frac{K}{H_{\alpha}} & =\sum_{k \in \mathcal{K}} \frac{1}{\alpha_{k}^{2}}, \\
C T & =\sum_{k \in \mathcal{K}} \log \left(\frac{P_{T}}{K \sigma^{2}}\right)=K \log \left(\frac{P_{T}}{K \sigma^{2}}\right) .
\end{aligned}
$$

On the other hand, at high SNR the sum rate of the UPA $R_{T}^{U P A}$ can be approximated as

$$
\begin{aligned}
R_{T}^{U P A} & \approx \sum_{k \in \mathcal{K}} \log \left(\frac{P_{T}}{\sigma^{2}} \frac{\alpha_{k}^{2}}{K}\right)=C T+\log \prod_{k \in \mathcal{K}} \alpha_{k}^{2}= \\
& =C T+K \log G_{\alpha},
\end{aligned}
$$

where $G_{\alpha}$ is the geometric mean of the $\alpha_{k}^{2}$, i.e.

$$
G_{\alpha}=\left(\prod_{k \in \mathcal{K}} \alpha_{k}^{2}\right)^{1 / K}
$$

which means that at high SNR the sum rate of the UPA $\left(R_{T}^{U P A}\right)$ is always greater or equal to the sum rate of the $\operatorname{ERB}\left(R_{T}^{E R B}\right)$. This comes from the fact that the geometric mean is always greater or equal than the harmonic mean, i.e.

$$
G_{\alpha} \geq H_{\alpha} \Rightarrow R_{T}^{U P A} \geq R_{T}^{E R B}
$$

in which equality holds when the $\alpha_{k}$ are all the same. This will unlikely occur in this case, because of the distributed nature of the users.

\section{APPENDIX II}

\section{EQUIVALENCE OF UPA AND MSR AT HIGH SNR}

The MSR is equivalent to the UPA if the SNR is high, since if $\sigma^{2}$ is low, then $\beta_{k}^{2} \approx \mu^{-1}$. Applying the constraint in (12), the power allocation reduces to $\beta_{k}^{2}=\frac{P_{T}}{K}$. If the number of users $K$ is low, or if the SNR is very high, all of them might be allocated for transmission. Under these circumstances, it is easy to verify that the power factors tend to be those from UPA using a result in [19]: if we let $Q$ and $K$ grow without bound, but their ratio $\zeta=Q / K$ remains fixed, then [19]

$$
\lim _{K, Q \rightarrow \infty} \mathbb{E}\left\{\frac{1}{\alpha_{k}^{2}}\right\}=\frac{1}{Q-K} .
$$

Introducing (13) into (12) to obtain $\mu^{-1}$ using the expression in (19), and then substituting $\mu^{-1}$ back into (13), the power allocation factors reduce to $\mathbb{E}\left\{\beta_{k}^{2}\right\}=\frac{P_{T}}{K}$. Therefore, at high SNR, the MSR tends to the UPA. Besides, since it has been proven that the UPA outperforms the ERB in terms of sum rate, the MSR provides also a higher sum rate than the ERB. However, the distribution of the resources is asymmetric and some users are given more resources than others.

\section{APPENDIX III}

\section{EQUIVALENCE OF ERB AND MSB AT HIGH SNR}

This might have computational implications, since the MSB is more complex than the ERB. First, note that if all the users in the active set $\mathcal{K}$ are served, $\log \mu$ in (16) can be calculated as

$$
\log \mu=\frac{\sum_{j \in \mathcal{K}} \frac{\sigma^{2}}{c_{2} \alpha_{j}^{2}} \log \left(c_{1} c_{2} \frac{\alpha_{j}^{2}}{\sigma^{2}}\right)-P_{T}}{\sum_{j \in \mathcal{K}} \frac{\sigma^{2}}{c_{2} \alpha_{j}^{2}}},
$$

so at high SNR, the first term in the numerator tends to zero, and the power allocation factor can then be approximated as

$$
\left|\beta_{k}\right|^{2} \approx \frac{\sigma^{2}}{c_{2} \alpha_{k}^{2}}\left(\log \left(c_{1} c_{2} \frac{\alpha_{k}^{2}}{\sigma^{2}}\right)+\frac{P_{T}}{\sum_{j \in \mathcal{K}} \frac{\sigma^{2}}{c_{2} \alpha_{j}^{2}}}\right) .
$$

At a high SNR, the linear term grows faster than the logarithmic term, so these power allocation factors reduce to

$$
\beta_{k}^{2} \approx \frac{\sigma^{2}}{c_{2} \alpha_{k}^{2}} \frac{P_{T}}{\sum_{j \in \mathcal{K}} \frac{\sigma^{2}}{c_{2} \alpha_{j}^{2}}}=\frac{P_{T}}{\alpha_{k}^{2}} \frac{1}{\sum_{j \in \mathcal{K}} \frac{1}{\alpha_{j}^{2}}}=\frac{P_{T}}{\alpha_{k}^{2}} \frac{1}{\operatorname{tr}\left(\mathbf{H} \mathbf{H}^{H}\right)^{-1}}
$$

which leads to a SNR for the $k$ th user given by

$$
\gamma_{k}=\frac{\alpha_{k}^{2} \beta_{k}^{2}}{\sigma^{2}}=\frac{P_{T}}{\sigma^{2}} \frac{1}{\operatorname{tr}\left(\mathbf{H H}^{H}\right)^{-1}},
$$

which is the same as in (10) for the ERB. Both BER methods tend to attain the same performance at high SNR, but note that this convergence does not occur with the rate-based schemes.

\section{APPENDIX IV \\ FAIRNESS ANALYSIS OF THE UPA, THE ERB, AND THE EPS}

In the analysis, we note that the term $\gamma^{n}$ is disregarded since it is a common factor of the three techniques, and the cardinality of the set $\mathcal{K}$ is $\mathrm{K}$. The objective is to compute a figure similar to that showing the measure of inequality called the Gini plot. Therefore, a maximum, mean, and minimum analysis among the users is required, which constitutes a reasonable approximation.

First, an analysis of the mean SNR is conducted. For the UPA, since the $\alpha_{k}^{2}$ behave like central Chi-squared random variables with $2(Q-K+1)$ degrees of freedom, where each random variable has variance $1 / 2$ i.e. $\alpha_{k}^{2} \sim \chi_{2(Q-K+1)}^{2}$, the mean value is given by

$$
\mathbb{E}\left\{\frac{\alpha_{k}^{2}}{K}\right\}=\frac{Q-K+1}{K} .
$$

Using a result of large random matrices, it has been already shown that for the ERB

$$
\mathbb{E}\left\{\frac{1}{\operatorname{tr}\left[\left(\mathbf{H H}^{H}\right)^{-1}\right]}\right\}=\frac{Q-K}{K},
$$


whereas for the EPS, the detailed analysis is given in [2]. The final result is that the mean value for the EPS can be approximated as

$$
\mathbb{E}\left\{\frac{\left\|\mathbf{h}_{k}\right\|^{2}}{\delta^{-1}}\right\} \approx \frac{Q}{Q-1} \frac{Q-K}{K}
$$

Since $\frac{Q}{Q-1}>1$ the mean value for the EPS is always greater than for the ERB in (25). On the other hand, after some algebra, it can be verified that if $K-1>0$, the EPS yields always a lower mean than the UPA in (24). Note that if $K=1$, then the three methods yield the same mean values. Therefore, the EPS yields a mean value which is in between the concern about the global performance (the UPA at high SNR) and the fulfillment of the individual needs (the ERB). The results in this section are in fact the diversity order of the proposed techniques, so the diversity order of the UPA is higher than that of the ERB, see (24) and (25), and the diversity order of the EPS is in between both methods. After that, it shall be proven that the dispersion between the maximum and minimum values is lower than for the UPA. The analysis will only be performed for the UPA and for the EPS, because the maximum and minimum values among the users are the same as the mean for the ERB, for which all the users are granted the same fraction of the resource, recall (25).

The procedure to obtain the behavior of the maximum or the minimum of any of the techniques is based on the statistical behavior of the SNR random variables. After some algebra, they are computed numerically in [2], thus we do not go into details in this Appendix.

\section{REFERENCES}

[1] P. Viswanath, D. N. C. Tse, and R. Laroia, "Opportunistic beamforming using dumb antennas," IEEE Trans. Inf. Theory, vol. 48, no. 6, pp. 12771294, June 2002.

[2] D. Bartolome, "Fairness analysis of wireless beamforming schedulers," PhD Dissertation, Jan. 2005, available at http://www.tdx.cesca.es/TDX0302105-134045/.

[3] R. W. Heath, M. Airy, and A. J. Paulraj, "Multiuser diversity for MIMO wireless systems with linear receivers," in Proc. 35th Asilomar Conference on Signals, Systems and Computers, Nov. 2001.

[4] M. Bengtsson and B. Ottersten, "Optimal and suboptimal transmit beamforming," in Handbook of Antennas in Wireless Communications, L. Godara, Ed. CRC Press, 2001.

[5] M. Bengtsson, D. Bartolomé, J. L. Vicario, and C. Antón-Haro, "Beamforming and bit-loading strategies for multi-user downlink SDMA with admission control," (invited paper), in Proc. IEEE PIMRC, Sept. 2005.

[6] H. Zhu and K. J. R. Liu, "The use of diversity antennas in high-speed wireless systems: capacity gains, fairness issues, multi-user scheduling," Bell Labs Technical Memorandum, 2001, available at http://mars.belllabs.com $/ \mathrm{cm} / \mathrm{ms} /$ what $/ \mathrm{mars} /$ papers/borst_whiting.

[7] J. Y. Le Boudec and B. Radunovic, "A unified framework for max-min and min-max fairness with applications," Proc. 40th Annual Allerton Conference on Communication, Control, and Computing, Oct. 2002.

[8] $\mathrm{K} . \mathrm{Xu}$, "How has the literature on Gini's Index evolved in the past 80 years?" Dalhousie University, Economics Working Paper., Apr. 2003, available from http://papers.ssrn.com/sol3/papers.cfm?abstract_id=423200.

[9] Z. Dongmei, X. Shen, and J. W. Mark, "Efficient call admission control for heterogeneous services in wireless mobile ATM networks," IEEE Commun. Mag., vol. 38, no. 10, pp. 72-78, Oct. 2000.
[10] D. Bartolomé and A. I. Pérez-Neira, "Practical implementation of bit loading schemes for multi-antenna multi-user wireless OFDM systems," submitted to IEEE Trans. Commun., Oct. 2004, revised Apr. 2005.

[11] S. Thoen, L. Van der Perre, M. Engels, and H. De Man, "Adaptive loading for OFDM/SDMA-based wireless networks," IEEE Trans. Commun., vol. 50, no. 11, pp. 1798-1810, Nov. 2002.

[12] G. Dimic and N. D. Sidiropoulos, "Low-complexity downlink beamforming for maximum sum capacity," Proc. International Conference on Acoustics, Speech, and Signal Processing (ICASSP), May 2004.

[13] D. Bartolome and A. I. Perez-Neira, "Performance analysis of scheduling and admission control for multiuser downlink SDMA," Proc. International Conference on Acoustics, Speech, and Signal Processing (ICASSP), May 2004.

[14] S. T. Chung and A. J. Goldsmith, "Degrees of freedom in adaptive fodulation: a unified view," IEEE Trans. Commun., vol. 49, no. 9, pp. 1561-1571, Sept. 2001.

[15] J. M. Cioffi, "A multicarrier primer," ANSI Doc., T1E1.4 Tech. Subcommittee, no. 91-157, 1991.

[16] D. Bartolome, A. Pascual Iserte, and A. I. Perez-Neira, "Spatial scheduling algorithms for wireless systems," Proc. International Conference on Acoustics, Speech, and Signal Processing (ICASSP), Apr. 2003.

[17] D. Piazza and L. B. Milstein, "Multiuser diversity-mobility tradeoff modeling and performance analysis of a proportional fair scheduling," Proc. Global Telecommunications Conference (GLOBECOM), Nov. 2002

[18] S. Boyd and L. Vanderberghe, Convex Optimization. Available online at http://www.stanford.edu/ boyd/bv_cvxbook.pdf, 2003.

[19] B. Hochwald and S. Wishwanath, "Space-time multiple access: linear growth in the sum rate," Proc. 40th Allerton Conference on Communications, Control and Computing, Oct. 2002.

[20] L. Zheng and D. N. C. Tse, "Diversity and multiplexing: a fundamental tradeoff in multiple antenna channels," IEEE Trans. Inf. Theory, vol. 49 , no. 5, pp. 1073-1096, May 2003.

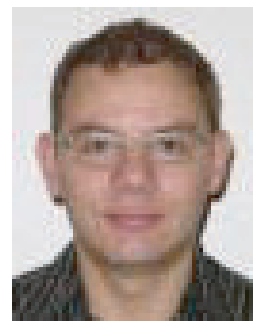

Diego Bartolomé was born in Barcelona, Spain, in 1977. He graduated in telecommunication engineering in 2000 , and he received his $\mathrm{PhD}$ in January 2005 from the Technical University of Catalonia (UPC), Barcelona, Spain. Moreover, he obtained his M.Sc. in Management and Business Administration in June 2005 from the Autonomous University of Barcelona (UAB). In 2003, he was granted by the Telecommunications Technological Center of Catalonia (CTTC) for the completion of his $\mathrm{PhD}$, which dealt about fairness issues in multi-antenna multiuser communications. He has actively participated in Spanish projects, as well as European projects (IST and Eureka) and Networks Of Excellence, where he contributed with his view on fairness issues in several physical layer mechanisms, e.g. multi-antenna techniques, power control, and bit allocation. $\mathrm{He}$ is the author of five journal papers and 18 conference papers. Currently, he is part of the Technology Transfer Area in the CTTC, Castelldefels, Spain.

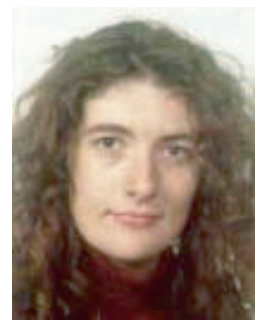

Ana I. Pérez-Neira was born in Zaragoza, Spain, in 1967. She graduated in telecommunication engineering in 1991 and received the Ph.D. degree in 1995 from the Universitat Politècnica de Catalunya (UPC), Barcelona, Spain. In 1991, she joined the Department of Signal Theory and Communication of the UPC, where she carried on research activities in the field of higher order statistics and statistical array processing. In 1992, she became Lecturer, and since 1996, she has been an Associate Professor at UPC, where she teaches and co-ordinates graduate and undergraduate courses in statistical signal processing, analog and digital communications, mathematical methods for communications and nonlinear signal processing. She is the author of three book chapters, 15 journal and more than 80 conference papers in the area of statistical signal processing and fuzzy processing, with applications to mobile/satellite communication systems, physical and access layers. She has coordinated national public and private founded projects and has participated in INTAS, RACE, ACTS, IST and Eureka European projects. She is IEEE Senior member. 